Proc. IEEE Trans. Nucl. Sci.

November $8-14,1998$

BNL -66889

Toronto, Canada

\title{
Two Dimensional Studies of Dynamics of Electron Clouds in Silicon Drift Detectors
}

\author{
R.Bellwied ${ }^{d}$, R.Beuttenmuller ${ }^{a}$, N.Brandon ${ }^{b}$ H.Caines $^{b}$, W.Chen ${ }^{a}$, D.DiMassimo ${ }^{a}$, H.Dyke ${ }^{b}$, \\ G.W.Hoffmann ${ }^{c}$, T.J.Humanic ${ }^{b}$, A.I.Kotova ${ }^{b}$, I.V.Kotov ${ }^{b, e, 1}$, H.W.Kraner ${ }^{a}$, Z.Lid ${ }^{a}$, D.Lynn ${ }^{a}$, G.Ott ${ }^{c}$, \\ S.U.Pandey ${ }^{d}$, C.Pruneau ${ }^{d}$, V.L.Rykov ${ }^{d}$, J.Schambach ${ }^{c}$, J.Sedlmeir $^{a}$, E.Sugarbaker ${ }^{b}$ J.Takahashi $^{d}$, \\ W.K.Wilson ${ }^{d}$
}

STAR-SVT Collaboration

a Brookhaven National Laboratory, Upton, NY 11973, USA

${ }^{b}$ The Ohio State University, Columbus, OH 43210, USA

${ }^{c}$ University of Texas Austin, Austin, TX 78712, USA

${ }^{d}$ Wayne State University, Detroit, MI 48201, USA

${ }^{e}$ IHEP, RU-148284 Protvino, Moscow region, Russia

\begin{abstract}
The dynamics of electrons generated in silicon drift detectors is studied using an IR LED. Electrons were generated at different drift distances. In this way the evolution of the cloud in anode and drift directions as a function of drift time was measured. For the anode direction the method of cumulative functions was used to extract the electron cloud profiles. The cloud width was obtained also from measurements of the charge collected on a single anode as a function of the coordinate of the light spot. We present the first report of the experimental measurements of the cloud width in drift direction extracted from signal waveforms. The evolution of the electron cloud width with drift time is compared with theoretical calculations. Theoretical expectations agree with our experimental results.
\end{abstract}

\section{Introduction}

In silicon drift detectors (SDDs) [1,2] fabricated on $\mathrm{n}$-type silicon the electrons created by an ionizing particle are forced by an electric field to drift toward readout anodes. The electric field inside SDDs is a superposition of a depletion field created by positive charges uniformly distributed in the depleted silicon bulk and a transport field created by a distribution of potentials on the cathodes. The depletion field is responsible for both the collection of electrons at the bottom of the potential valley and the confinement of the created electron cloud in the middle of the bulk during the drift. Along the drift path the cloud expands, due to such effects as diffusion and mutual electrostatic repulsion between electrons. The diffusion itself causes an increase of the cloud width $\sigma(t)=\sqrt{2 D t}$, where $t$ is the drift time and $\mathrm{D}$ is the diffusion coefficient. Broadening of the pulses from SDDs affects the coordinate and double particle resolution as well as design parameters of front end electronics. A theoretical analysis of the dynamics of electrons is presented in [3] for the case of a constant transport field.

\footnotetext{
${ }^{1}$ Corresponding author. Phone: (614)-292-4775; fax: (614)-292-4833; e-mail: kotov@mps.ohio-state.edu
}

In this paper we present experimental results on the dynamics of electron clouds in SDDs. Electrons were generated at different drift distances and the shape of the cloud arriving on the anodes was measured. In this way the cloud shape dependence on drift distance was studied. Measurements were done using a STAR2.9 detector. This is a bidirectional SDD designed for the silicon vertex tracker of the RHIC STAR experiment [4]. Maximum drift distance is $30 \mathrm{~mm}$. Detector thickness is $300 \mu \mathrm{m}$. Further details about STAR SDDs can be found in $[5,6]$.

Section 1 briefly describes the experimental setup. In section 2 the methods used to measure the cloud shape are discussed. Section 3 shows experimental results.

\section{Experimental Setup}

For these measurements detectors were bonded to mother boardis with preamplifier sockets and bias voltages. The front end electronics consisted of charge-sensitive hybrid preamplifiers and hybrid shapers [7]. The data were read out via a $500 \mathrm{MHz}$ digital oscilloscope TDS640A.

An IR LED [8] of wavelength $820 \mathrm{~nm}$ was used to generate electrons. The repetition rate was $300 \mathrm{~Hz}$, the duration of a light pulse less than $8 \mathrm{~ns}$. At $820 \mathrm{~nm}$ an initial charge is generated near the detector surface. The LED light was focused, by microscope, into a spot of about $20 \mu m$ in diameter. Light pulses were transported to the microscope via an optical fiber. The spot was positioned in the $35 \mu \mathrm{m}$ gaps between cathodes. One light pulse generated about $6.2 \times 10^{4}$ electrons, i.e about 2.5 times that of a minimum ionizing particle crossing the detector plane. Visible light was used to check the focusing at different drift distances.

The SDD was placed under the microscope on a XY stage with a position resolution of $0.5 \mu \mathrm{m}$ for both axes. The oscilloscope and controller of the XY stage were connected via GPIB to a Macintosh computer. Software for automatization of measurements was witten using LabVIEW [9]. 
We acquired average waveforms of signals from three adjoining anodes at different positions of the light spot along the "drift" axis $(\mathrm{X})$ and the axis parallel to the anodes ( $\mathrm{Y}$ ). Measurements were performed in eleven positions along drift length. At each drift distance the light spot was moved along the $\mathrm{Y}$ axis with a step of $25 \mu \mathrm{m}$ and $40-65$ measurements were taken. An example of waveforms taken closest to the anode position is shown in Fig. $\frac{1}{12}$.
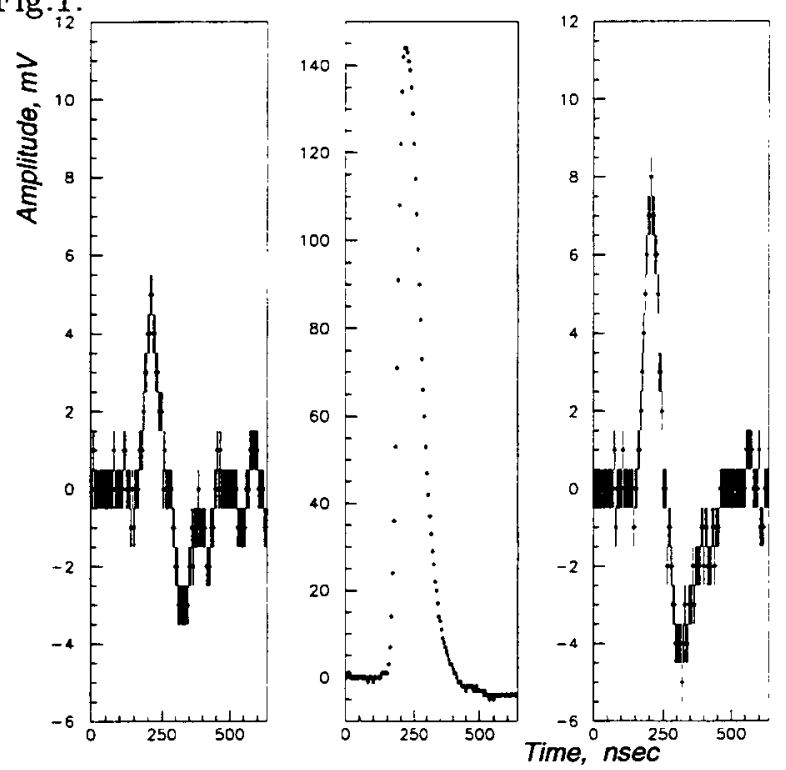

Figure 1: Waveforms from three anodes at "zero" drift distance and collection of total generated charge on the central anode.

The transport field was $400 \mathrm{~V} / \mathrm{cm}$. The drift time versus drift distance is shown in Fig.2. The drift velocity is $5.2 \mu \mathrm{m} / \mathrm{ns}$.

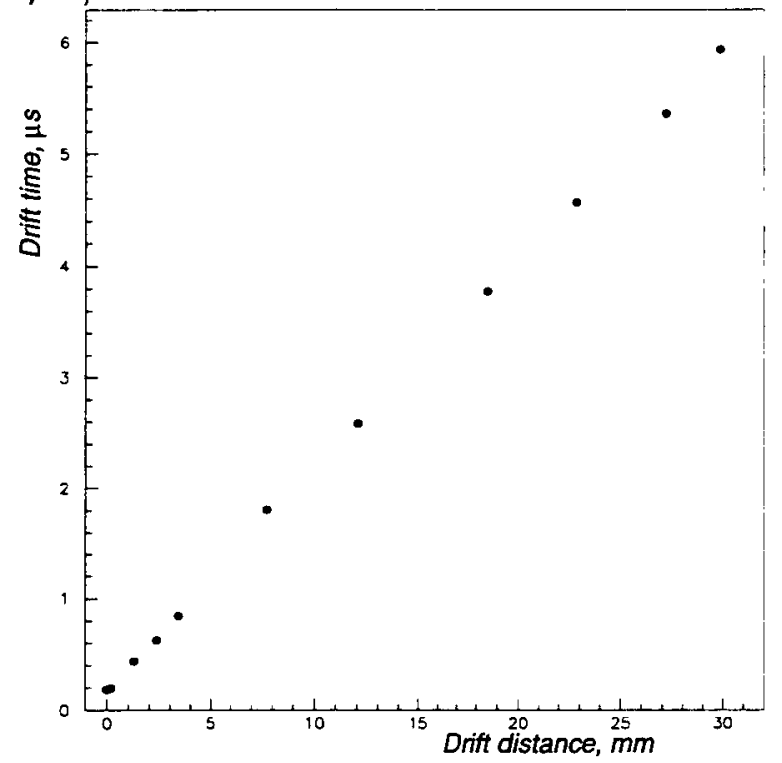

Figure 2: Drift time vs. drift distance.

\section{Methods}

\section{A. Anode direction}

Our goal is to determine the charge density of the electron cloud projected onto the $\mathrm{X}$ or $\mathrm{Y}$ axis (i.e. the "profile") for a cloud drifted time $t$ from the point of generation to the readout anodes.

Let $y$ be the distance from the cloud center to the edge of the readout anode, $Q_{\text {total }}$ the total charge of the cloud and $n(y, t)$ normalized density. The charge collected at this individual anode is

$$
Q(y)=Q_{\text {total }} \times \int_{y}^{y+\Delta} n\left(y^{\prime}, t\right) d y^{\prime},
$$

where $\Delta$ is the effective anode length along $\mathrm{Y}$ axis. For our detector $\Delta$ is $250 \mu \mathrm{m}$. If the anode dimension is larger than the cloud size or we sum over enough anodes to cover the cloud we can rewrite (1) in terms of the cumulative function $\Phi(y, t)$

$$
\Phi(y, t)=\frac{Q(y)}{Q_{\text {total }}}=\int_{y}^{\infty} n\left(y^{\prime}, t\right) d y^{\prime} .
$$

Moving the light spot along $\mathrm{Y}$ we can directly measure the cumulative function $\Phi(y, t)$. The derivative of the $\Phi(y, t)$ with respect to $y$ gives the average electron cloud profile $n(y, t)=\partial \Phi(y, t) / \partial y$. It is worth mentioning that the same technique is applicable to studies of electromagnetic showers as demonstrated in [10]. Factors limiting the accuracy of measurements are pulse-to-pulse fluctuations of LED, electronic noise, accuracy in positioning of the XY stage and precision of the calibration. The contribution of all statistical fluctuations decreases with increasing statistics. As long as position resolution is much smaller than the cloud width the related error is small.

As will be shown clouds expand beyond three anodes at large drift times. So, it is important to have another method for estimating the cloud size. A method for estimating the cloud size using measurements on a single anode is shown, below. Assume that the cloud profile is gaussian-like. The charge collected at an individual anode is

$$
Q(y)=Q_{\text {total }} \times\left[F R E Q\left(\frac{y+\Delta}{\sigma}\right)-F R E Q\left(\frac{y}{\sigma}\right)\right],
$$

where $F R E Q$ is the frequency function defined in [11]. Measuring the charge collected at the anode as a function of coordinate of the light spot and applying standard fitting technique the cloud width and total charge can be obtained. Obviously this method is free of systematic errors related to calibration.

\section{B. Drift direction}

For analysis in the drift direction we use the fact that the output signal from the anode is a convolution of an 
electronics response function with the anode signal. In Laplace representation, the electronics response function for our preamplifiers and shapers is

$$
F(s)=\frac{s}{\left(s+\frac{1}{\tau_{s}}\right)^{3}\left(s+\frac{1}{\tau_{l}}\right)},
$$

where $\tau_{s}$ and $\tau_{l}$ are two electronics constants. As will be shown the shape of the electron cloud in the anode direction is Gaussian. So, by fitting signal waveforms with a convolution of the electronics response function and a Gaussian, we are able to extract the cloud width in the drift direction.

Analytical expression for this convolution was obtained and coded as a FORTRAN function. To determine the electronics parameters $\tau_{s}$ and $\eta_{n}$, waveforms were initially fitted using five free parameters $\tau_{s}, \tau_{l}, t_{0}, \sigma, Q$, where $t_{0}$ and $\sigma$ are the position and width of the Gaussian respectively, and $Q$ is the charge collected on that anode. $\tau_{s}$ and $\pi_{l}$ were then fixed at average values obtained from about 200 waveforms taken at different drift distanses.

The anode signal induced by this Gaussian cloud will have some deviations from a Gaussian shape, but we expect that those deviations are not significant.

\section{Experimental results}

\section{A. Anode direction}

The method of cumulative functions was used to extract the cloud shape for those drift times where the cloud is fully covered by three anodes. In Fig.3 the measured fractions of the collected charge, $Q(y) / Q_{\text {total }}$, as a function of $y$ are shown for the light spot position closest to the anodes. At that drift distance each anode provides two measurements of the cumulative function. The left edge gives $\Phi(\hat{y})$ and the right edge gives $1-\Phi(\hat{y})$ according to the definition (2) of the cumulative function. Differentiation of measured functions gives $n(y, t)$ and $-n(y, t)$ separated by the distance $\Delta$. Fig.4 shows cloud profiles calculated by numerical differentiation of $Q(y) / Q_{\text {total }}$. The profiles have a Gaussian-like form. The width of the cloud at this point is $\sigma \simeq 20 \mu \mathrm{m}$ in agreement with the light spot size.

For all drift distances the amplitude from a single anode measured as a function of coordinate of the light spot were fitted by Eq. 3 with free parameters $Q_{t o t a l}$ and $\sigma$. Results of the fit for all drift distances for one anode are shown in Fig.5. The quality of the fit is good for all distances. Both methods are in good agreement.

The evolution of the cloud width with drift time is shown in Fig.6. The dynamics of the electrons is described by the continuity equation [3]. The solution of the continuity equation, which takes into account drift, diffusion and Coulomb repulsion, for $Q=6.2 \times 10^{4}$ electrons is shown by a solid line. Values of other parameters used in calculations are: diffusion coefficient
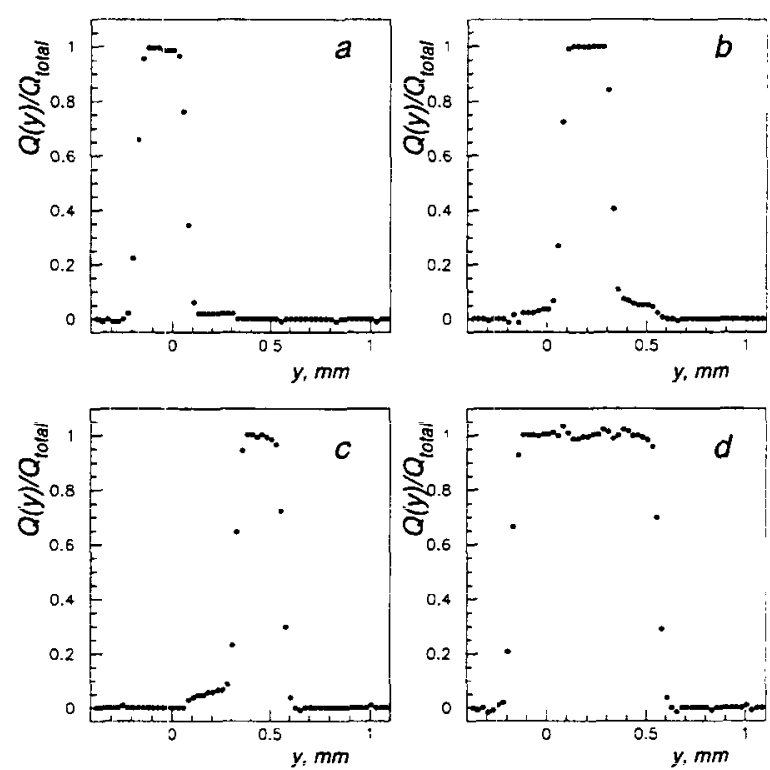

Figure 3: Fraction of the charge collected on the anode(s) vs. position of the light spot. a), b), c) - - individual anodes; d) - sum over three anodes.
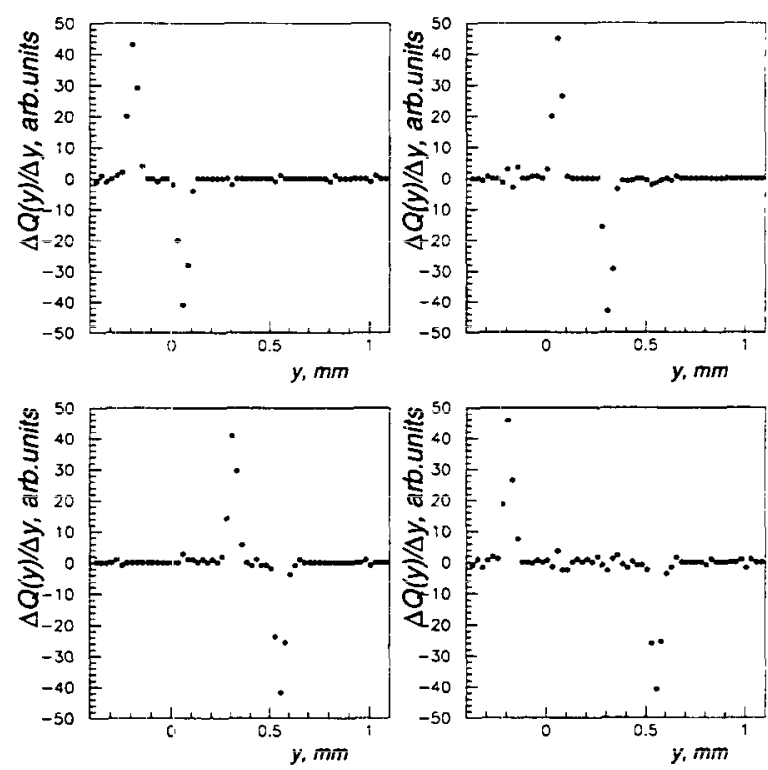

Figure 4: Cloud profiles.

$D=3.5 \mu \mathrm{m}^{2} / n s$, electron mobility $\mu=1350 \mu \mathrm{m}^{2} / n s \dot{V}$. The agreement between measurements and theoretical model of the dynamics of electrons is evident. The dash line shows the evolution of the cloud width because of the diffusion only. Dotted line shows the evolution of the cloud width for the case of the Coulomb repulsion only. Even at this low ionization, the contribution of Coulomb repulsion is significant, particularly at short times while the cloud size is small $(\leq 100 \mu m)$.

\section{B. Drift direction}

Results of the waveform fits for one anode at four drift distances are shown in Fig.7. The quality of fits is good at all drift distances. 

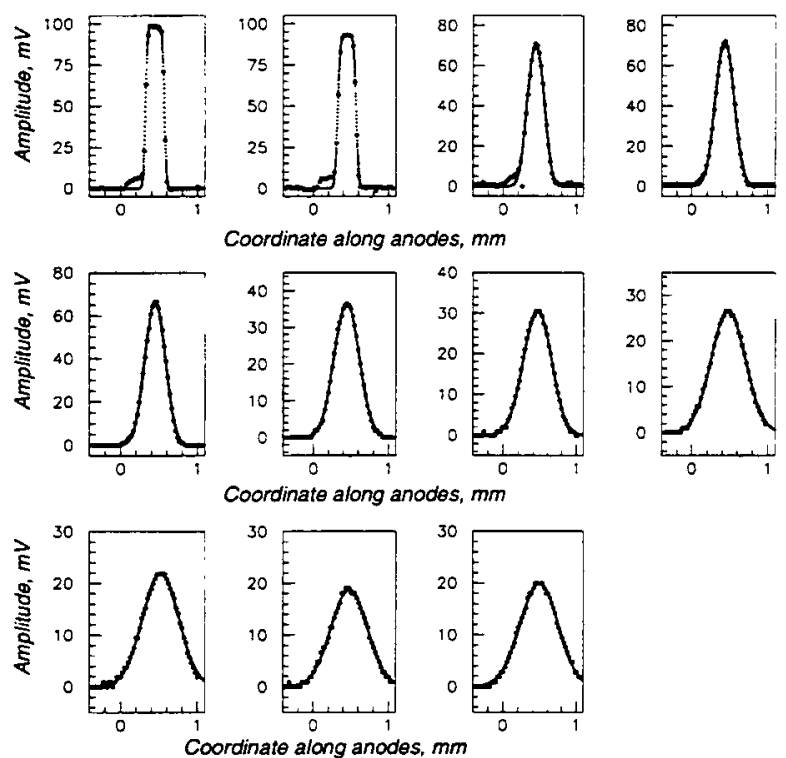

Figure 5: Amplitudes from a single anode vs light spot coordinate for all drift distances going from $0 \mathrm{~mm}$ to 29.89 $\mathrm{mm}$ in sequence: $0.0,0.18,1.26,2.35,3.42,7.74,12.07,18.55$, $22.87,27.20,29.89 \mathrm{~mm}$. Points are data, solid line is fit.

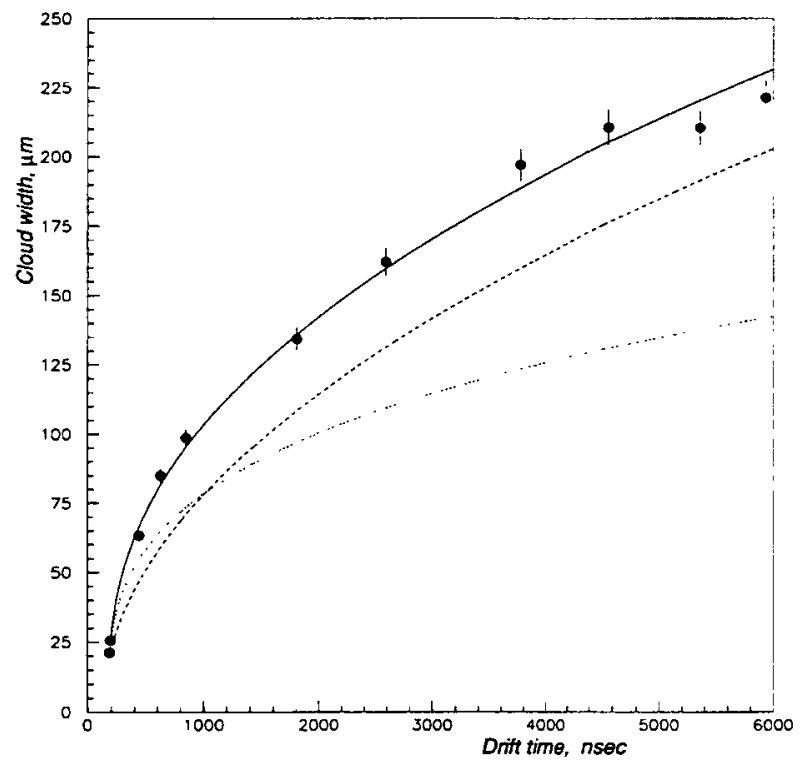

Figure 6: Dependence of the cloud size on drift time. Points are data. Solid line shows a solution of the continuity equation with both diffusion and Coulomb repulsion contributions. Dash line shows the diffusion only. Dotted line shows Coulomb repulsion only.

For each drift distance the width $\sigma$ of the Gaussian was extracted from waveforms taken at different coordinates of the LED spot along the anode. For the first three drift distances we see a parabolic dependence of $\sigma$ on the coordinate along the anode with a minimum at the center of the anode. At very short drift distances, the electron cloud expands rapidly. Even a small variation in drift distance will cause visible variation in the cloud size. This could be a possible explanation of the effect. The width of the cloud for the first three distances was determined
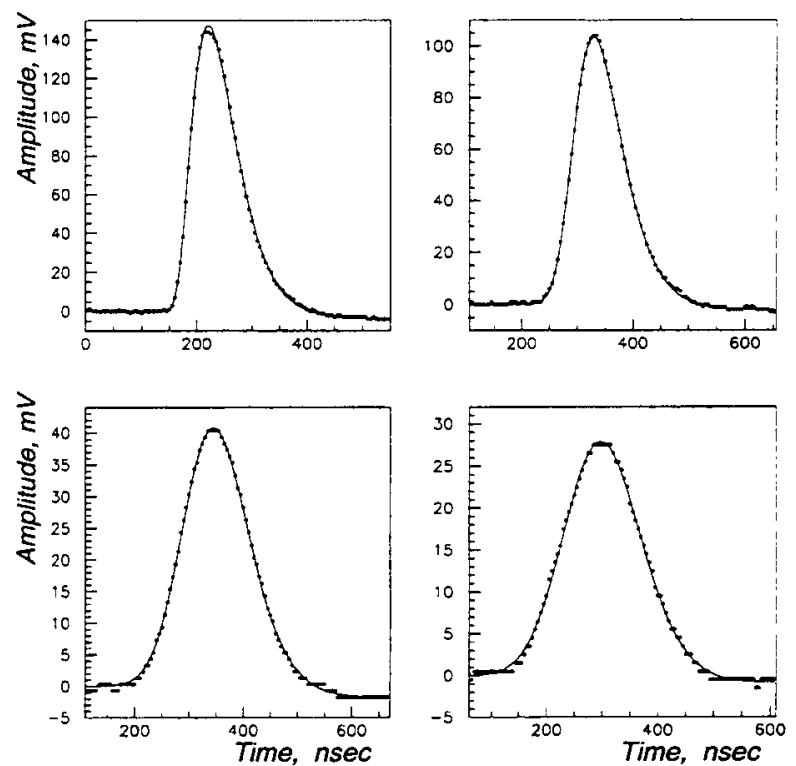

Figure 7: Signal waveforms for drift distances $0 ., 2.35,12.07$, $29.89 \mathrm{~mm}$. Black dots are data, solid lines show best fits.

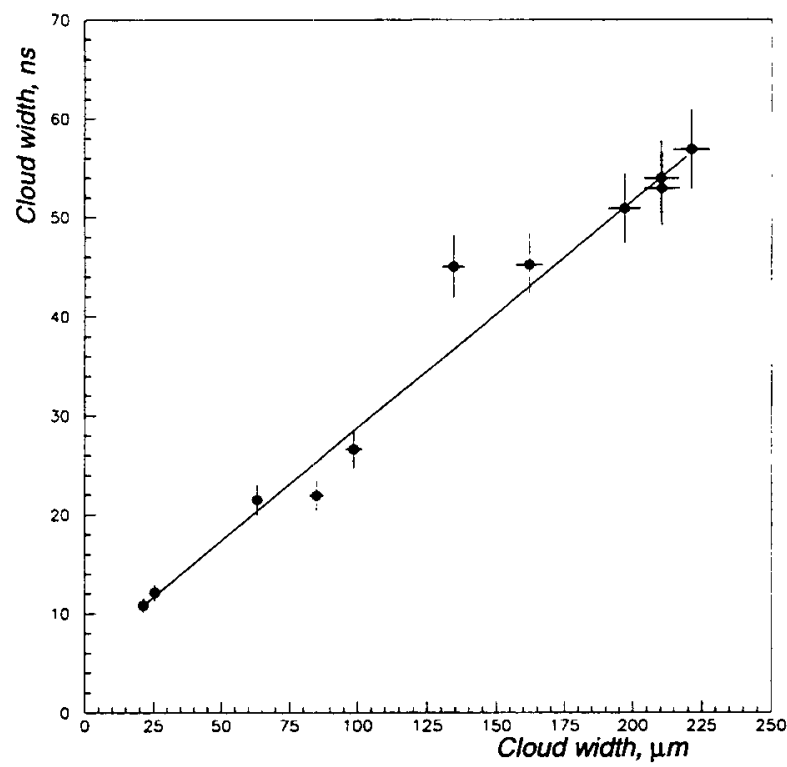

Figure 8: Cloud width in drift direction vs. cloud width in anode direction. Black dots are data, solid line shows linear dependence.

as the value of $\sigma$ in the minimum, for other distances the weighted average was used.

A correlation between cloud width in anode and drift directions is shown in Fig.8. It is a linear dependence. That confirms that in both directions the electron cloud expands in the same way. As one can see from Fig. 8 the size of the cloud in the drift direction is systematically larger than the size in the anode direction. For our measurements this can be explained by the following: 1) the duration of the LED pulse increases the size of the initial spot only in the drift direction; 2) the signal induced on the anode by a single electron is a function with a finite width; 3 ) the drift field is not uniform in the focusing region and 
4) there are small local non uniformities in the drift field outside the focusing region. Taking that into account we can conclude that the theoretical model [3] of the dynamics of electrons in silicon drift detectors is in agreement with our experimental results.

\section{Acknowledgments}

We thank D.Fiske for his help in data analysis. This work was supported in part by the US Department of Energy grants DE-AC02-98CH10886 and DE-FG0394ER-40485, NSF grant PHY-9803859, STAR R\&D funds and Robert A. Welch Foundation.

\section{References}

[1] E.Gatti and P. Rehak, Nucl. Inst. and Meth. 225 (1984) 608.

[2] P.Rehak et al., Nucl. Inst. and Meth. A235 (1985) 224234.

[3] E.Gatti, A.Longoni, P. Rehak et al., Nucl. Inst. and Meth. A253 (1987) 393-399.

[4] J.W.Harris and the STAR collaboration. Nucl. Phys. A566 (1994) 277c-286c.

[5] R.Bellwied et al., Nucl. Inst. and Meth., A377 (1996) 387-392.

[6] R.Bellwied et al., Nucl. Inst. and Meth., A400 (1997) 279-286.

[7] Veljko Radeka, Low-noise techniques in detectors, Ann. Rev. Nucl. Part. Sci. 1988, 38 p.217-277.

[8] HFBR-1202 Transmitter, Hewlett Packard Company, Optoelectronics Designer's Catalog 1988-89, p.8-78.

[9] LabVIEW 3.1 National Instruments.

[10] C.Cerri et al., Nucl. Inst. and Meth., 214 (1983) 217235.

[11] Normal Frequency Function, CERNLIB - CERN Program Library Short Writeups, CERN Geneva, Switzerland, p. C301-1, May 1995 
